Draft VERsion June 9, 2021

Preprint typeset using $\mathrm{IAT}_{\mathrm{E}} \mathrm{X}$ style emulateapj v. 5/2/11

\title{
STELLAR VELOCITY DISPERSION MEASUREMENTS IN HIGH-LUMINOSITY QUASAR HOSTS AND IMPLICATIONS FOR THE AGN BLACK HOLE MASS SCALE
}

\author{
C. J. Grier ${ }^{1}$, P. Martini ${ }^{1,2,3}$, L. C. Watson ${ }^{4}$, B. M. Peterson ${ }^{1,2}$, M. C. Bentz $^{5}$, K. M. Dasyra ${ }^{6}$, M. Dietrich $^{7}$, \\ L. Ferrarese ${ }^{8}$, R. W. Pogge ${ }^{1,2}$, \& Y. $\mathrm{ZU}^{1}$ \\ Draft version June 9, 2021
}

\begin{abstract}
We present new stellar velocity dispersion measurements for four luminous quasars with the NIFS instrument and the ALTAIR laser guide star adaptive optics system on the Gemini North 8-m telescope. Stellar velocity dispersion measurements and measurements of the supermassive black hole masses in luminous quasars are necessary to investigate the coevolution of black holes and galaxies, trace the details of accretion, and probe the nature of feedback. We find that higher-luminosity quasars with higher-mass black holes are not offset with respect to the $M_{\mathrm{BH}}{ }^{-} \sigma_{*}$ relation exhibited by lower-luminosity AGNs with lower-mass black holes, nor do we see correlations with galaxy morphology. As part of this analysis, we have recalculated the virial products for the entire sample of reverberation-mapped AGNs and used these data to redetermine the mean virial factor $\langle f\rangle$ that places the reverberation data on the quiescent $M_{\mathrm{BH}}{ }^{-} \sigma_{*}$ relation. With our updated measurements and new additions to the AGN sample, we obtain $\langle f\rangle=4.31 \pm 1.05$, which is slightly lower than, but consistent with, most previous determinations.

Subject headings: galaxies: active — galaxies: kinematics and dynamics — galaxies: nuclei — quasars: individual (PG 1411+442, PG 1617+175, Mrk 509, PG 2130+099)
\end{abstract}

\section{INTRODUCTION}

Over the past couple of decades, both observational and analytical work have suggested a physical connection between the formation and growth of galaxies and the growth of their central black holes. For example, the comoving emissivity of active galactic nuclei (AGNs) and the cosmic star formation rate have both similarly declined since $z \sim 1$ (Boyle et al. 1998; Franceschini et al. 1999; Merloni et al. 2004; Silverman et al. 2008), which might imply a link between star formation and AGN activity. In addition, luminous AGNs are more often found in massive early-type galaxies with young stellar populations (e.g., Sanders et al. 1988; Kauffmann et al. 2003; Veilleux et al. 2009). Further support of a black holegalaxy connection comes in the form of a number of correlations between properties of the host galaxies and the masses of their central black holes (BHs). A key relationship is between black hole mass $\left(M_{\mathrm{BH}}\right)$ and bulge stellar velocity dispersion $\left(\sigma_{*}\right)$, observed in both quiescent (Ferrarese \& Merritt 2000; Gebhardt et al. 2000a; Tremaine et al. 2002; Gültekin et al. 2009; McConnell et al. 2011; McConnell \& Ma 2013) and active galaxies (Gebhardt

\footnotetext{
${ }^{1}$ Department of Astronomy, The Ohio State University, 140 W 18th Ave, Columbus, OH 43210, USA

${ }^{2}$ Center for Cosmology and AstroParticle Physics, The Ohio State University, Columbus, OH 43210, USA

${ }^{3}$ Visiting Astronomer, North American ALMA Science Center and University of Virginia, Charlottesville, VA 22903, USA

${ }^{4}$ Harvard-Smithsonian Center for Astrophysics, 60 Garden Street, Cambridge, MA 02138, USA

${ }^{5}$ Department of Physics and Astronomy, Georgia State University, Atlanta, GA 30303, USA

6 Observatoire de Paris, LERMA (CNRS:UMR8112), 61 Av. de l'Observatoire, F-75014, Paris, France

${ }^{7}$ Department of Physics and Astronomy, Ohio University, Athens, OH 45601, USA

${ }^{8}$ Herzberg Institute of Astrophysics, National Research Council of Canada
}

et al. 2000b; Ferrarese et al. 2001; Nelson et al. 2004; Onken et al. 2004; Dasyra et al. 2007; Woo et al. 2010; Graham et al. 2011; Park et al. 2012). This relation was first predicted by Silk \& Rees (1998) and Fabian (1999) and has been explained by various analytic models (e.g., King 2003; King 2005; Murray et al. 2005) as well as recovered in numerical simulations of evolving and interacting galaxies (e.g., Di Matteo et al. 2005, 2008). The $M_{\mathrm{BH}}-\sigma_{*}$ relationship can be used to infer $M_{\mathrm{BH}}$ in large samples of galaxies. This allows for the exploration of the $\mathrm{BH}$ mass function on much larger scales (e.g., Yu \& Tremaine 2002) and thus helps investigate the role of $\mathrm{BHs}$ in galaxy formation and evolution processes.

Direct $M_{\mathrm{BH}}$ measurements are made with stellar kinematics and gas dynamics, although these methods require good spatial resolution and are presently only feasible for nearby galaxies. AGNs, however, offer the most robust tracer of the evolution of the $\mathrm{BH}$ population over much of the history of the universe. Under the assumption that the motion of the gas in the broad line region (BLR) of AGNs is dominated by the gravitational influence of the black hole, one can use the virial relation $M_{\mathrm{BH}}=\left(f R_{\mathrm{BLR}} \Delta V^{2}\right) / G$ to obtain $M_{\mathrm{BH}}$, where $R_{\mathrm{BLR}}$ is the average radius of the emitting gas in the BLR, usually either determined with reverberation mapping (e.g., Peterson et al. 2004) or estimated with the radiusluminosity relation (e.g., Bentz et al. 2009a, 2013), $\Delta V$ is the velocity dispersion of the gas, deduced from the width of the emission line, and $f$ is a dimensionless factor that accounts for the unknown geometry and orientation of the BLR and may be different for each AGN.

With current technology, we are unable to directly observe the structure of the BLR, as it is unresolvable even with the largest telescopes, so the true value of $f$ for each object is unknown. This has contributed significantly to the uncertainties in $M_{\mathrm{BH}}$ measurements using BLR emis- 
sion lines. Recent reverberation mapping (RM) efforts have begun to reveal more information about the actual structure of the BLR and the value of $f$ in some objects (e.g., Bentz et al. 2010; Brewer et al. 2011; Pancoast et al. 2012; Grier et al. 2013). However, limited data for most AGNs requires the use of an average virial factor $\langle f\rangle$ to estimate $M_{\mathrm{BH}}$. Currently, $\langle f\rangle$ is calculated with the assumption that AGNs follow the same $M_{\mathrm{BH}}-\sigma_{*}$ relation as quiescent galaxies (Onken et al. 2004; Woo et al. 2010; Graham et al. 2011; Park et al. 2012; Woo et al. 2013). Most estimates of $\langle f\rangle$ are somewhat larger than $\sim 5$; Onken et al. (2004) find $\langle f\rangle=5.5 \pm 1.8$, Woo et al. (2010) find $\langle f\rangle=5.2 \pm 1.2$, and more recently, analysis by Park et al. (2012) and Woo et al. (2013) both yield $\langle f\rangle=5$.1. Graham et al. (2011) obtain a slightly lower value, $\langle f\rangle=3.8_{-0.6}^{+0.7}$

The difference between slopes and virial factors among studies using similar regression methods (whether $M_{\mathrm{BH}}$ is considered the independent or dependent variable) arise when different galaxy samples are used to determine these two quantities, which may suggest a morphological dependence or selection bias in the relation. In fact, recent studies do report a morphological dependence in the quiescent $M_{\mathrm{BH}}-\sigma_{*}$ relation, such that there are systematic differences in the relation for early-type (highermass) and late-type (lower-mass) galaxies (e.g., Greene et al. 2010; McConnell \& Ma 2013). Others have found that barred galaxies lie systematically below the $M_{\mathrm{BH}^{-}}$ $\sigma_{*}$ relation of normal unbarred galaxies (e.g., Graham 2008a,b; Graham \& Li 2009), and still others have found deviations in both the slope and intercept for galaxies hosting pseudobulges (e.g., Hu 2008; Gadotti \& Kauffmann 2009; Kormendy et al. 2011). The idea of a nonuniversal $M_{\mathrm{BH}^{-}}-\sigma_{*}$ relation has been supported by theoretical work as well (e.g., King 2010; Zubovas \& King 2012), which has also suggested that the relation may depend on environment.

Morphological deviations from a single $M_{\mathrm{BH}}-\sigma_{*}$ relation have also been claimed in AGNs (e.g., Graham \& Li 2009; Mathur et al. 2012), and there has been some question as to whether or not objects at the high-mass/high$\sigma_{*}$ end of the relation follow a different slope (e.g., Dasyra et al. 2007; Watson et al. 2008). For example, four out of the six objects with $M_{\mathrm{BH}}$ above $10^{8} M_{\odot}$ included in the study of Watson et al. (2008) lie significantly above the relation. The appearance of outliers could be due to systematic errors in $\sigma_{*}$ or $M_{\mathrm{BH}}$ measurements, or simply a fluke due to small number statistics. Alternatively, Lauer et al. (2007) suggest that offsets at the high-mass end may be due to a selection bias. Specifically, when a sample is selected based on AGN properties, one is more likely to find a high-mass $\mathrm{BH}$ in a lower-mass galaxy (based on a $\mathrm{BH}$-host galaxy correlation) because high mass galaxies are rare and there is intrinsic scatter in $\mathrm{BH}$-host galaxy correlations.

An important step in evaluating the $M_{\mathrm{BH}}-\sigma_{*}$ relation and any possible deviations from it is to obtain secure $\sigma_{*}$ and $M_{\mathrm{BH}}$ measurements in AGNs that sample the entire mass range of the relation. While the highmass end of the quiescent $M_{\mathrm{BH}}-\sigma_{*}$ relation is relatively well-populated to beyond $10^{9} M_{\odot}$ (McConnell \& Ma $2013)$, the current sample of AGNs used to calculate $\langle f\rangle$ still contains just three or four objects with $M_{\mathrm{BH}}$ above
$10^{8} M_{\odot}$ (Graham et al. 2011; Park et al. 2012; Woo et al. 2013). More measurements for luminous AGNs are needed to measure the high-mass end of the AGN $M_{\mathrm{BH}}-\sigma_{*}$ relation. However, accurate $\sigma_{*}$ measurements for high-luminosity AGNs are difficult to obtain because the AGN light overpowers the light from the host. Moreover, more luminous AGNs are relatively scarce and thus typically found at large distances, so the host galaxy has a small angular size and is easily lost in the glare of the AGN. It is only in the past few years that highprecision measurements in very luminous objects have been obtained on account of the availability of adaptive optics (AO) and integral field spectrographs (IFUs) such as Gemini North's Near-Infrared Integral Field Spectrometer (NIFS) combined with the Gemini North laser guide star AO system, ALTAIR. Watson et al. (2008) used NIFS+ALTAIR to measure $\sigma_{*}$ for PG $1426+015$ with much higher precision than previous measurements for high-luminosity quasars. This success prompted us to undertake additional observations of quasars at the high-mass end of the $M_{\mathrm{BH}}-\sigma_{*}$ relation. In this paper we present the results of our NIFS observations of eight high-luminosity quasars. We successfully measured $\sigma_{*}$ in four objects and use these results to improve the population of the $M_{\mathrm{BH}}-\sigma_{*}$ relation at the high-mass end. We also recalculate virial products for the entire AGN sample with updated time lag measurements to re-derive $\langle f\rangle$, calibrate black hole masses in AGNs, and reexamine the AGN $M_{\mathrm{BH}^{-}} \sigma_{*}$ relation. In this work we adopt a cosmological model of $\Omega_{m}=0.3, \Omega_{\Lambda}=0.70$, and $H_{0}=70 \mathrm{~km}$ $\mathrm{sec}^{-1} \mathrm{Mpc}^{-1}$.

\section{OBSERVATIONS AND DATA ANALYSIS}

\subsection{NIFS/ALTAIR Observations}

Observations of eight quasars were carried out at the Gemini North telescope in 2008 and 2010 under the programs GN-2008B-Q-28, GN-2010A-Q-11, and GN2010B-Q-24. We chose our sample from the database of objects with RM-based black hole mass measurements from Peterson et al. (2004) with $M_{\mathrm{BH}}>10^{8} M_{\odot}$. Basic information on our targets is given in Table 1 . We used NIFS in conjunction with the ALTAIR laser guide star AO system to carry out our observations. NIFS has a $3^{\prime \prime} \times 3^{\prime \prime}$ field of view that is divided into 29 individual spectroscopic slices, with a spectral resolution $R=$ $\lambda / \Delta \lambda \approx 5290$ in both the $H$ and $K$ bands. With the AO correction, NIFS yields a spatial resolution on the order of $0.1^{\prime \prime}$. There are several strong stellar absorption lines that fall within the wavelength range of the $H$ band, which has a central wavelength of $1.65 \mu \mathrm{m}$ and covers from about $1.49 \mu \mathrm{m}$ to $1.80 \mu \mathrm{m}$, so we observed seven of our targets with the $H$ band filter. We list the most prominent stellar absorption features in this wavelength region in Table 2 . We observed our eighth object, PG $1700+518$, in the $K$ band due to its higher redshift. The $K$ filter on NIFS covers from about $1.99 \mu \mathrm{m}$ to 2.40 $\mu \mathrm{m}$.

We estimated the integration time for each object with $H S T$ ACS or WFPC2 images of the sources from Bentz et al. (2009a). To simulate the data we would obtain from NIFS, we measured the flux within a $3^{\prime \prime} \times 3^{\prime \prime}$ aperture, except for a central circle of diameter $0.2^{\prime \prime}$. We estimated the exposure time for each object based on its 

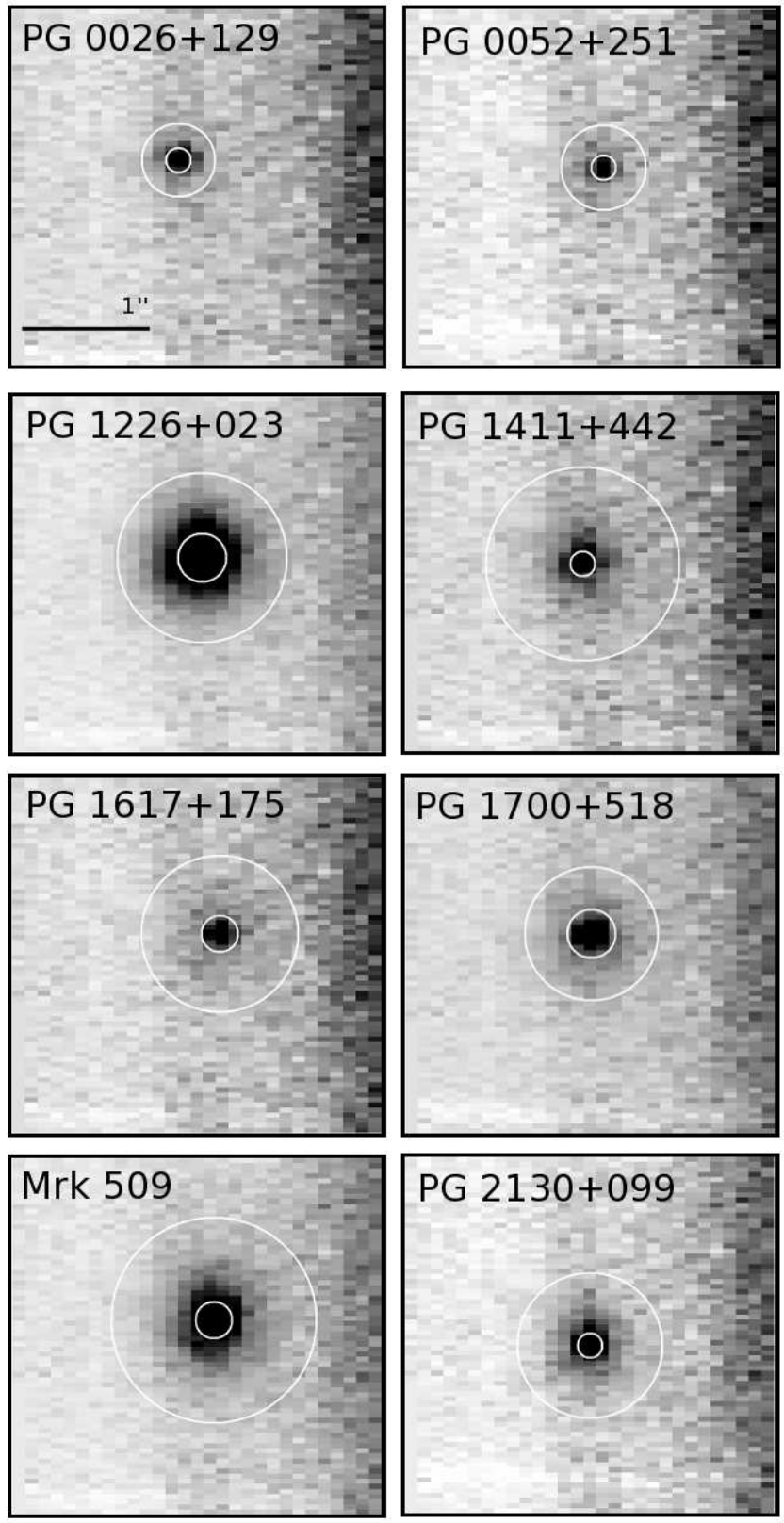

Fig. 1.- Raw reconstructed images for each object. The white circles denote the extraction annuli used for each object, given in Table 4 . The field of view of each panel is $3^{\prime \prime} \times 3^{\prime \prime}$.

brightness relative to PG $1426+015$, for which Watson et al. (2008) obtained a host-galaxy signal-to-noise ratio $(S / N) \sim 200$ in about two hours of on-source integration. With both Poisson and background-limited trials, we estimated the integration time required for each object to obtain a $S / N \sim 200$. Table 3 gives details of the observations, most notably the on-source integration time for each object. Reconstructed images from the IFU spectra of all eight targets are shown in Figure 1. We observed telluric standard stars (usually A0V) once every 1.5 hours for the purpose of telluric corrections.

\subsection{Data Reduction}

Data were processed through the standard NIFS pipeline $^{9}$ from the Gemini IRAF ${ }^{10}$ package. Our reductions deviated from the standard pipeline tasks in only two ways. First, we found that the original sky frames did not adequately remove sky lines from our spectra. To remedy this, we manually scaled the individual sky spectra to obtain better sky subtraction in each individual object frame. Second, to remove stellar absorption lines in our telluric spectra, we used methods described by Vacca et al. (2003) and applied in the IDL-based code xtellcor. This code uses a theoretical model of Vega to remove the hydrogen features in our telluric standard spectra, and is specifically written for use with A0V stars.

To separate the host-galaxy spectra from the AGNs, we extract the spectrum from an annulus that excludes the quasar-dominated nucleus. The use of AO in these observations allowed us in most cases to confine at least the core of the quasar flux to the very central pixels of the image. Generally, the AO-assisted seeing was on the order of $0.1-0.2^{\prime \prime}$, so we used either a $0.2^{\prime \prime}, 0.3^{\prime \prime}$, or $0.4^{\prime \prime}$ inner radius $\left(R_{\text {inner }}\right)$ to isolate the quasar component. The outer radius for each extraction annulus $\left(R_{\text {outer }}\right)$ was chosen to include as much host galaxy light as possible while minimizing the amount of noise contributed by the sky. For most of our objects, we evaluated this by eye and chose windows that minimized noise. However, in the targets where we could see identifiable galactic absorption lines, we chose $R_{\text {inner }}$ and $R_{\text {outer }}$ to obtain the highest equivalent width measurements in the visible absorption lines. $R_{\text {inner }}$ and $R_{\text {outer }}$ for each object are listed in Table 4 and are shown on the reconstructed images in Figure 1. The total galaxy+quasar spectra for all eight objects, extracted from within a radius of $R_{\text {outer }}$, are shown in the top panels of Figure 2.

Although the use of the ALTAIR AO system helps confine the nuclear light to the central few pixels of the image, the AO-corrected PSF still has nuclear light in its wings. Typical Strehl ratios for the ALTAIR AO system are on the order of 0.1 to 0.3 in the $H$ band (Christou et al. 2010). For a typical Strehl ratio of 0.2, Christou et al. (2010) report a 50\% encircled PSF energy radius of about $0.4^{\prime \prime}$; i.e., half of the quasar light falls in a radius outside $0.4^{\prime \prime}$. Thus, there is still significant quasar contamination in the host-galaxy spectra. To further remove the quasar emission, we scaled and subtracted the nuclear spectrum from our annulus in each target. The scaling was done empirically — we chose a scale factor that best eliminated the most prominent quasar emission lines seen in the spectra. Our final nucleus-subtracted, observed-frame spectra of all eight objects are shown in the bottom panels of Figure 2. Note that while the spectrograph coverage in the $H$ band extends from about 1.48 $\mu \mathrm{m}$ to $1.8 \mu \mathrm{m}$, in some cases the telluric contamination was sufficiently significant that we cropped the spectra before using them. The wavelength coverage of the $H$ band spectra shown in Figure 2 is not uniform for this reason.

\footnotetext{
9 http://www.gemini.edu/sciops/instruments/nifs

10 IRAF (Tody 1986) is distributed by the National Optical Astronomy Observatories, which are operated by the Association of Universities for Research in Astronomy, Inc., under cooperative agreement with the National Science Foundation.
} 
PG $0026+129$

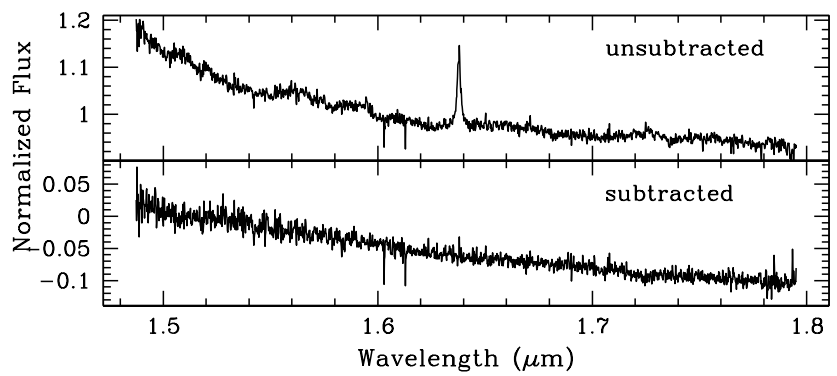

PG $0052+251$

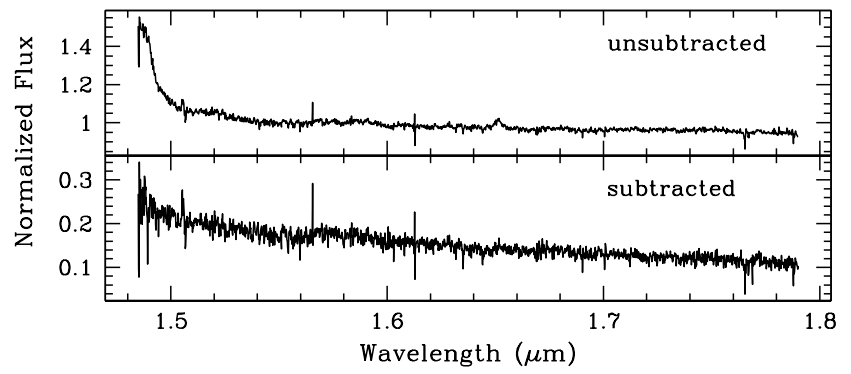

PG $1226+023$

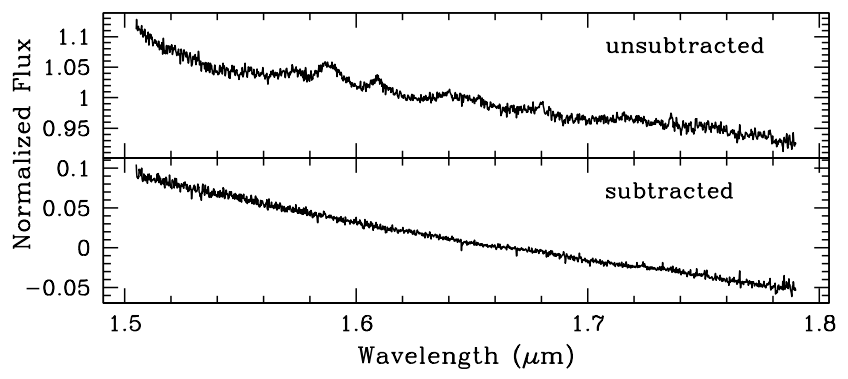

PG $1411+442$

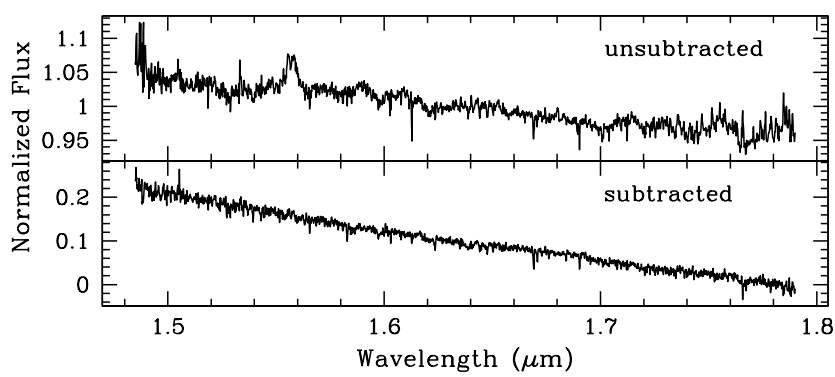

FIG. 2.- Observed-frame spectra of all eight targets. The top panels show the total, unsubtracted spectrum, and the bottom panels show the host galaxy spectrum after the nucleus was subtracted off. The fluxes are in units of flux per unit wavelength, and have been normalized to the mean of the unsubtracted spectrum for each object.

\subsection{Stellar Velocity Dispersion Measurements}

We used the penalized pixel fitting method (pPXF) of Cappellari \& Emsellem (2004) to measure $\sigma_{*}$. This method convolves a stellar template spectrum and a lineof-sight velocity distribution to model the host galaxy. The best line-of-sight velocity distribution is calculated in the pPXF code with a $\chi^{2}$ minimization technique. The velocity templates used to make our measurements were obtained by Watson et al. (2008), and include stars of four different spectral classes: K0 III, K5 III, M1 III, and

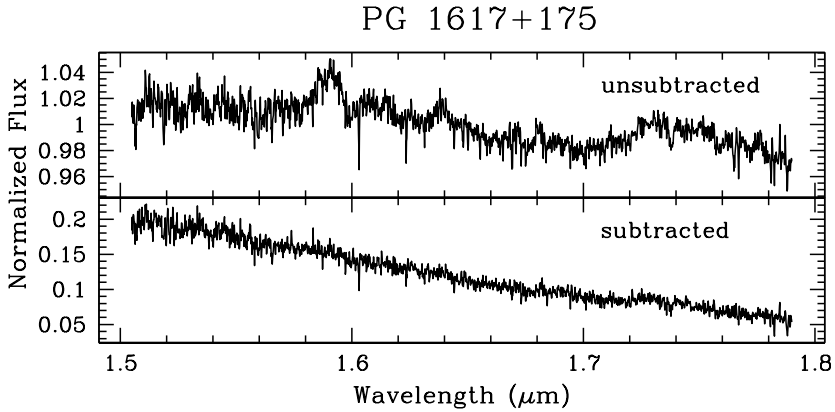

PG $1700+518$

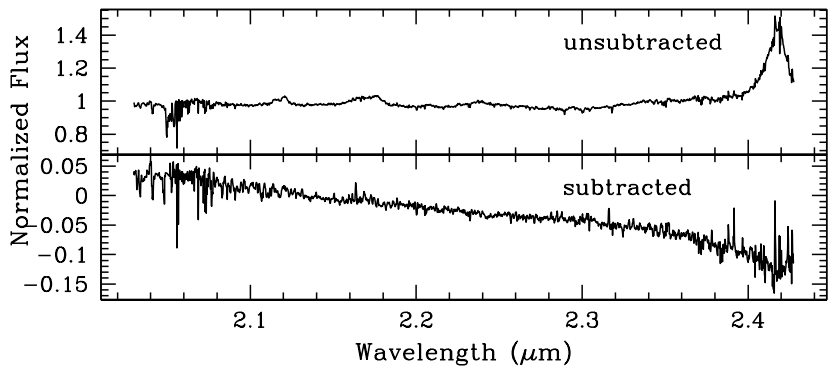

Mrk 509

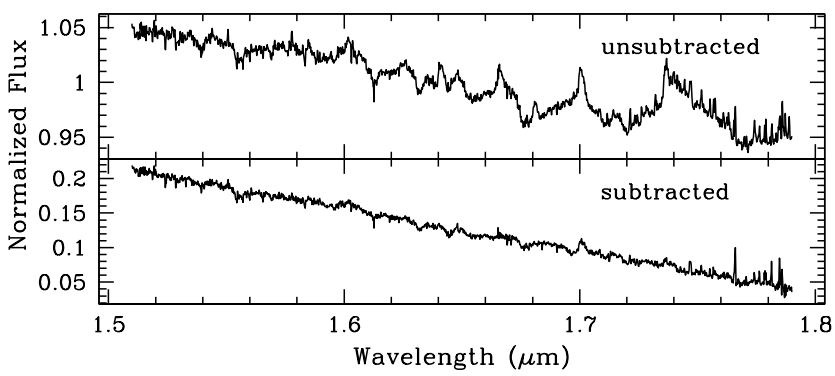

PG $2130+099$

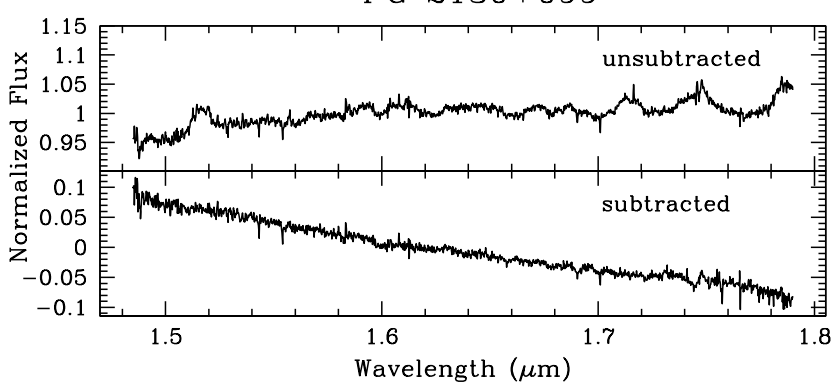

FIG. 2.- Continued.

M5 Ia. The K5 III, M1 III, and M5 Ia templates all resulted in somewhat similar fits in each spectrum - the reduced $\chi^{2}$ values of the fit in these three cases were always very close to one another, with the K5 III template usually a slightly better fit than the other two. The K0 III template provided a poor fit to the $\mathrm{CO}(3-0)$ absorption line in all objects and was therefore not used in our analysis.

There are several different factors that affect the uncertainty in $\sigma_{*}$. First, no single stellar template is expected to be a perfect match to the host-galaxy stellar absorption features. We therefore adopt the average $\sigma_{*}$ value from the three templates as our estimate, and fold the differences into our uncertainties. One exception to this is Mrk 509, for which the K5 III template provided a sig- 
nificantly better fit. We take the standard deviation in $\sigma_{*}$ reported by the pPXF software among the three stellar templates as representative of the template mismatch uncertainty. We also consider the location of $\Delta \chi^{2}=1$ in the fitting a component of our uncertainties. To do this, we allowed $\sigma_{*}$ to vary but held the rest of the parameters fixed at their best-fit values. We identified the value of $\sigma_{*}$ at which the $\chi^{2}$ had changed by \pm 1 from the best-fit value, and took the average difference between these two values and our best-fit value as our uncertainty. Because our measurements for PG $1411+442$ and PG $1617+175$ were made with spectra that are significantly noisier than the other two spectra, we also include a signal-to-noise $(S / N)$ component in our uncertainties for those two objects. To estimate this component, we degraded our two best spectra (Mrk 509 and PG 2130+099) to match the $S / N$ in $\mathrm{PG} 1617+175$ and $\mathrm{PG} 1411+442$. We then remeasured $\sigma_{*}$ in the degraded spectra and took the deviation from our original measurements to represent the $S / N$ component of the uncertainties. For most of the templates, the $\sigma_{*}$ measurements tended to be overestimated in the degraded spectra by about $20 \mathrm{~km} \mathrm{~s}^{-1}$. We combine the $1 \sigma$ uncertainties, the template mismatch uncertainties, and any $S / N$ component in quadrature and adopt these as our formal uncertainties.

We measured $\sigma_{*}$ from the spectra of four of our objects: Mrk 509, PG1411+442, PG 1617+175, and PG 2130+099, and these values are listed in Table 4 . The normalized galaxy spectra and the best-fit broadened stellar templates for these four objects are shown in Figure 3. Our measurement for PG 2130+099 of $147 \pm$ $17 \mathrm{~km} \mathrm{~s}^{-1}$ constitutes a significant improvement in precision over its previous measurement of $172 \pm 46 \mathrm{~km} \mathrm{~s}^{-1}$ (Dasyra et al. 2007), while Mrk 509 and PG 1411+442 have no previous measurements reported in the literature. Our measurement for PG $1617+175$ of $201 \pm 37$ $\mathrm{km}^{-1}$ is slightly more precise but consistent with measurements made by Dasyra et al. (2007), who report $\sigma_{*}$ $=183 \pm 47 \mathrm{~km} \mathrm{~s}^{-1}$. In the other four objects, we were unable to identify any absorption lines in our nucleussubtracted spectra and were unable to fit stellar templates and recover $\sigma_{*}$. We believe this was caused mainly by the overwhelming strength of the quasar emission in these objects and a lack of strong absorption lines within the observed wavelength range. These four objects are also the most distant of the eight we observed. In an attempt to minimize the quasar contamination, we experimented with different radii for both the inner and outer regions in these objects, but in all cases were unable to remove the quasar contamination enough to see absorption in the host galaxy spectrum. Even when subtracting off a scaled nuclear spectrum to eliminate the emission lines, we see only noise (with residual sky and telluric contamination; see Figure 2) in the spectra of objects in which we were unable to measure $\sigma_{*}$.

Because we optimized our extraction radii to obtain the best host-galaxy-to-quasar ratio, our $\sigma_{*}$ measurements were made within different effective physical apertures for each target. The measurements for Mrk 509, PG 1411+442, and PG 1617+175 were made within an effective physical aperture of $1.25 \mathrm{kpc}, 3.2 \mathrm{kpc}$, and 3.3 kpc respectively. The effective radii $\left(r_{\mathrm{e}}\right)$ for the spheroid components of these three objects from the surface de-
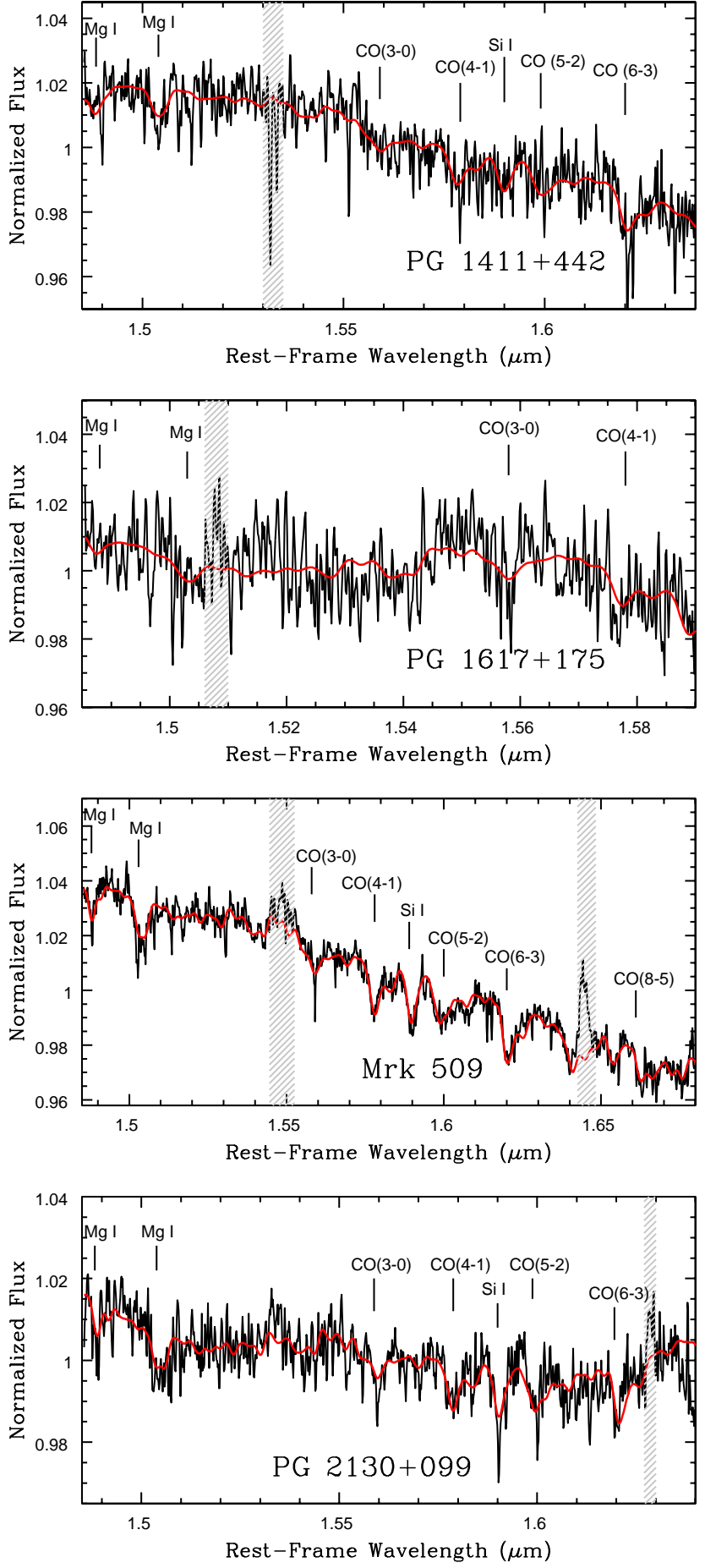

FIG. 3.- Normalized rest-frame spectra of objects in which we measured $\sigma_{*}$ successfully. The red lines are our best-fit models, with the K5 III stellar template for PG 1411+442, Mrk 509, and PG 2130+099, and the M5 Ia template for PG 1617+175. The shaded gray areas mark areas excluded from the fit due to telluric and/or quasar light contamination. 
compositions of Bentz et al. (2009a) are $1.85 \mathrm{kpc}, 5.05$ $\mathrm{kpc}$, and $3.3 \mathrm{kpc}$, so our apertures for these three objects lie between, or very close to, the commonly quoted aperture sizes of $r_{\mathrm{e}}$ and $r_{\mathrm{e}} / 8$. However, the measurement for PG 2130+099 was made within $1.64 \mathrm{kpc}$, which is 4.3 times $r_{\mathrm{e}}$ for this object. We apply the relation derived by Jorgensen et al. (1995) to determine the velocity dispersion within the effective radius $\left(\sigma_{*, \mathrm{e}}\right)$ for all four objects. These values are listed in Table 4 for comparison with our measurements. For Mrk 509, PG 1411+442, and PG $1617+175$, there is very little difference between our measured $\sigma_{*}$ and $\sigma_{*, \mathrm{e}}$. The value of $\sigma_{*, \mathrm{e}}$ for PG $2130+099$ is notably higher than our quoted value, but the change does not qualitatively affect our analysis. We use the corrected $\sigma_{*}$ measurements $\left(\sigma_{*, \mathrm{e}}\right)$ for the subsequent discussion and analysis.

\subsection{Recalculation of AGN Virial Products}

There are now 30 reverberation-mapped AGNs with $\sigma_{*}$ measurements. The virial products for most of these objects were calculated from time lags that were determined with traditional cross correlation methods (e.g., Peterson et al. 2004; Bentz et al. 2009c). However, Zu et al. (2011) recently introduced a different method to determine time lags that they called Stochastic Process Estimation for AGN Reverberation (SPEAR). This method works as follows: We assume all emission-line light curves are scaled and shifted versions of the continuum light curve. The continuum is modeled as an autoregressive process using a damped random walk (DRW) model, which has shown to be a good statistical model of quasar variability (Gaskell \& Peterson 1987; Kelly et al. 2009; Kozłowski et al. 2010; MacLeod et al. 2010; Zu et al. 2013). The transfer function is modeled as a simple top-hat function. We fit the continuum and emission-line light curves simultaneously, maximizing the likelihood of the model using Bayesian Markov Chain Monte Carlo iterations. The main advantage of the SPEAR method is that it treats gaps in the temporal coverage of light curves in a statistically self-consistent way, so the gaps in the data are filled in a well-defined manner with well-defined uncertainties.

$\mathrm{Zu}$ et al. (2011) re-measured the time lags in reverberation-mapped AGNs with the SPEAR method and demonstrated its ability to recover accurate time lags. The method has since been successfully used to improve RM measurements (Grier et al. 2012; Dietrich et al. 2012) and even recover velocity-delay maps (Grier et al. 2013). Because many of the light curves from the AGN $M_{\mathrm{BH}}-\sigma_{*}$ sample, particularly high-luminosity objects, have large gaps, we went through and re-determined the virial products for the entire sample with updated $\mathrm{H} \beta$ time lags recovered with the SPEAR method. Eighteen of the objects already have $\mathrm{H} \beta$ time lags from $\mathrm{Zu}$ et al. (2011). For eight of the remaining objects, we applied the same SPEAR method with the latest version of the software called JAVELIN ${ }^{11}$ to calculate new time lags and virial products. Three of the remaining AGNs have recently-published virial products calculated with the SPEAR method, so we use the published virial products for 3C 390.3 from Dietrich et al. (2012), 3C 120 from Grier et al. (2012), and PG 2130+099 from Grier et al.

\footnotetext{
$\begin{array}{ccc}11 & \text { Available at: } & \text { http://www.astronomy.ohio- } \\ \text { state.edu/ } \sim \text { yingzu/codes.html\#javelin }\end{array}$
}

(2013). We do not have the light curve for Mrk 50, so for this object we use the virial product from Barth et al. (2011). All recalculated time lags, original line widths, and updated virial products for each RM data set are given in Table 5. For objects with just one measurement, we use that virial product. For objects with multiple measurements, we adopt the mean of the logarithm of the virial products. In Table 6 we show the adopted virial products and $\sigma_{*}$ measurements for the entire sample.

In most cases, the updated virial products are very similar to the previously-quoted values. The median fractional change in the virial products due to the updated time lags is about $18 \%$, and the majority of these changes are consistent to within the measurement errors. For eight of the objects, the virial products changed somewhat significantly. The largest increase was for 3C 390.3, for which the new virial product increased by factor of almost five over the previous measurement. Most of these eight objects had light curves with significant gaps at key locations in the light curves that likely interfered with the time lag determinations. We do not see any systematic increase or decrease in the fractional change as a function of virial product (i.e., the virial products did not increase more in objects with larger virial products, or vice versa, in general), but we do note that the virial products of both of the highest-mass objects, 3C 390.3 and PG 1426+015, both increased substantially from previous measurements.

\section{RESULTS AND DISCUSSION}

\subsection{Data Quality}

The objects for which we successfully measure $\sigma_{*} \quad(\mathrm{PG} 1411+442, \quad \mathrm{PG} 1617+175, \quad$ Mrk 509, and PG 2130+099) are the four lowest-redshift galaxies in our sample. Mrk 509 and PG 2130+099 have the highest $S / N$ host-galaxy spectra, with an average $S / N$ per pixel of $\sim 250$ for Mrk 509 and $\sim 190$ for PG $2130+099$. These have the most easily identifiable galaxy absorption features in our sample (see Figure 3). The higher-luminosity quasars, PG 1411+442 and PG 1617+175, have lower $S / N$, specifically a $S / N$ of 100 per pixel in $\mathrm{PG} 1617+175$ and 130 per pixel in PG $1411+442$, which is lower than our anticipated $S / N$. Although we made several attempts to fit and remove sky features, residual sky contamination and telluric absorption lines remain in the subtracted spectra, which makes the velocity dispersion measurements more uncertain and contributes to the lower $S / N$ of the spectra. We also see stronger quasar emission features which we were unable to eliminate entirely from the host-galaxy spectra.

There seems to be three main factors that compromise the quality of our host galaxy spectra. First, we were unable to satisfactorily remove the sky emission from the spectra in all eight targets. This caused a significant decrease in $S / N$ in all of our spectra. Secondly, in three of our objects, PG 0026+129, PG 1126+023, and PG 0052+251, many of the strong stellar absorption features that allow us to measure $\sigma_{*}$ were redshifted out of the $H$-band. This limited us to very few absorption lines, and these few remaining lines fell in regions with severe telluric contamination. As such, we did not detect any absorption and were unable to measure $\sigma_{*}$ in these 
objects. Third, and possibly most importantly, in these four objects the quasar contamination becomes strong enough to overwhelm the host galaxy flux despite our long integrations and attempts to optimize the extraction radius. To quantify the amount of quasar contamination remaining in the original extraction annuli of these spectra, we estimated the ratio of the quasar flux to the host flux, both within our extraction radius, for the case of PG0026+129. We based this calculation on measurements of the PSF magnitude, host galaxy magnitude, and host galaxy Sersic index reported by Veilleux et al. (2009) from their analysis of $H S T$ NICMOS $H$-band images. We integrate the Sersic profile over the extraction annulus used in our study (for PG 0026+129, we used an inner radius of $0.2^{\prime \prime}$ and an outer radius of $0.6^{\prime \prime}$, corresponding to 0.62 and $1.86 \mathrm{kpc}$, respectively) to estimate the amount of host flux inside our aperture. Given the previously discussed findings of Christou et al. (2010), we assume that half of the PSF light falls inside the annulus and find that the PSF flux inside the extraction annulus is a factor of 4.5 times the amount of host flux inside the extraction annulus. We expect similar, possibly even more, contamination in the other targets for which we were unsuccessful, and thus this contamination limits our ability to explore the hosts of quasars at the high end of the luminosity distribution.

While we appear to have reached the limit of the NIFS+ALTAIR system for these measurements, AO systems continue to move towards diffractionlimited resolution with high Strehl ratios. These advances may lead to successful measurements with similar exposure times. The future availability of the James Webb Space Telescope (JWST) may also lead to successful attempts at $\sigma_{*}$ measurements in highluminosity quasars. JWST is currently expected to launch in 2018 and will be equipped with an IFU spectrograph of sufficient resolution, and the major problems of sky and telluric contamination, which were prohibitive for our higher-redshift targets, will be completely eliminated in space. Observing from space will allow us to see the whole spectrum continuously, so we will not be limited to specific redshift windows, and will also include the Ca II triplet region. $J W S T$ will offer a compact, stable PSF, and will have more sensitivity than our current equipment and thus offers much promise for future efforts to measure $\sigma_{*}$ in high-luminosity, high-redshift AGNs.

\subsection{The Faber-Jackson Relation}

It is also possible that the host galaxies of the four quasars in which we were unable to measure $\sigma_{*}$ were simply fainter galaxies with lower $\sigma_{*}$. If this is the case, excluding them in our subsequent examination of the $M_{\mathrm{BH}}-\sigma_{*}$ relation could result in a bias in the distribution at the high- $M_{\mathrm{BH}}$ end. As a separate check on the expected velocity dispersions of these hosts (as well as the rest of the sample), we place the entire RM sample on the Faber \& Jackson (1976) relation (hereafter the Faber-Jackson relation), which is the correlation between $\sigma_{*}$ and the absolute magnitude or luminosity of the host bulge. Some of the galaxies had already-published absolute $V$-band magnitudes from Bentz et al. (2009b), and we used host galaxy decompositions by Bentz et al. (2009a) and Bentz et al. (2013) to determine the bulge magnitudes in the rest (except Mrk 50, which was not in-

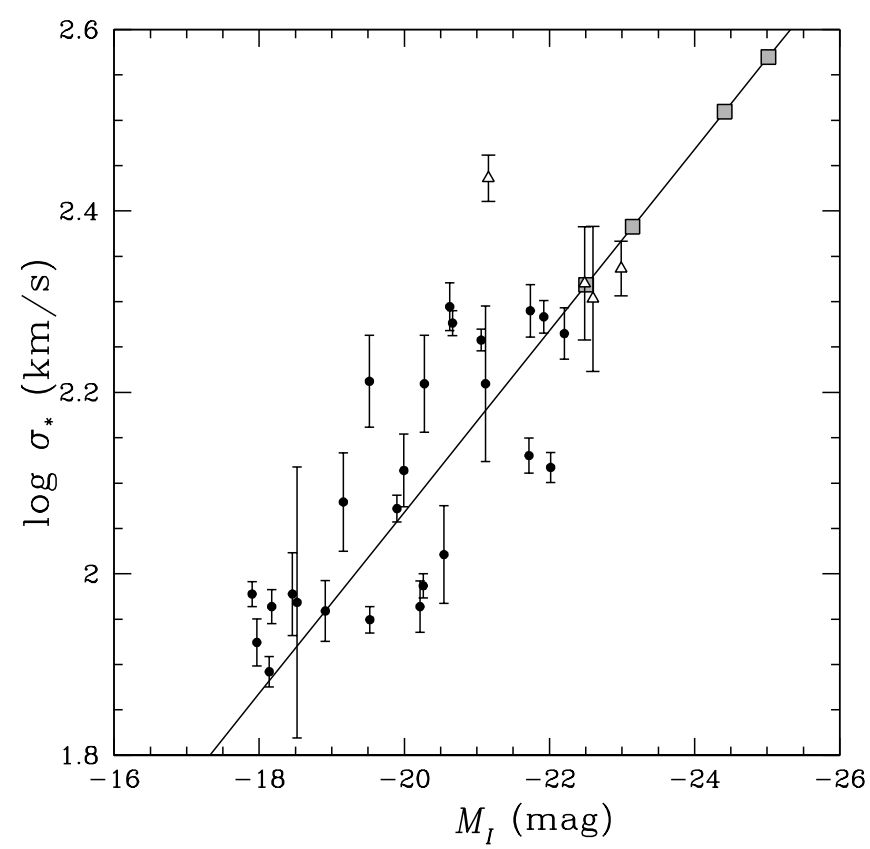

FIG. 4.- The RM sample on the Faber-Jackson relation. The solid black line shows the relation measured by Jiang et al. 2011 with the Gültekin et al. 2009 galaxy sample. Filled black circles show the RM sample with secure $\sigma_{*}$ measurements. Open triangles show the locations of objects with $M_{\mathrm{BH}}>10^{8} M_{\odot}$, and gray filled squares show the expected locations of the four targets in our study for which we were unable to obtain $\sigma_{*}$ measurements, based on the relation from Jiang et al. 2011.

cluded in these studies). To compare our sample with a previous determination of the Faber-Jackson relation by Jiang et al. (2011), we convert our magnitudes to the $I$ band. The HST observations (Bentz et al. 2009a, 2013) were taken using the ACS F550M filter and the WFPC2 F547M filter, while the images used by Jiang et al. (2011) were taken with the WFPC2 F814W filter. We use the IRAF package synphot with bulge templates from Kinney et al. (1996) to determine the $m_{F 550 M}-m_{F 814 W}$ and $m_{F 547 M}-m_{F 814 W}$ colors for each galaxy type and use this to transform our bulge magnitudes to the magnitude in the F814W filter, which is extremely close to the $I$ band.

Figure 4 shows our RM sample on the Faber-Jackson relation from Jiang et al. (2011), which was calculated with the sample from Gültekin et al. (2009). The RM sample as a whole appears to follow this relation, with some scatter. We use the same relation measured by Jiang et al. (2011) with the sample from Gültekin et al. (2009) to calculate the expected $\sigma_{*}$ values for the four objects in which we were unable to measure $\sigma_{*}$. As Figure 4 shows, the hosts of the four targets in wich we were unable to measure $\sigma_{*}$ are some of the most luminous in the sample, and thus the predicted $\sigma_{*}$ values are quite high. As such, we conclude that our inability to measure $\sigma_{*}$ in these objects is most likely due to the high AGN luminosities and high redshifts of the systems and not due to systematically fainter host galaxies in these targets. It is important again to note that measuring bulge properties in AGN, particularly at the high-luminosity end of the distribution, is very difficult, and thus the bulge luminosities themselves are subject to large uncertainties. 


\subsection{The Virial Factor $\langle\boldsymbol{f}\rangle$ and the $\boldsymbol{M}_{\mathbf{B H}}-\boldsymbol{\sigma}_{*}$ Relation}

Because we have updated the virial products in the AGN $M_{\mathrm{BH}}-\sigma_{*}$ sample and added a few objects at the high-luminosity end of the distribution, we also present an updated measurement of the average virial factor $\langle f\rangle$ used to calibrate the AGN $M_{\mathrm{BH}}$ scale. In order to measure $\langle f\rangle$, we assume that the AGN $M_{\mathrm{BH}^{-}} \sigma_{*}$ relation follows the same slope as quiescent galaxies. However, previous studies have found that AGNs appear to follow a slightly shallower relation than quiescent galaxies (e.g., Woo et al. 2010; Graham et al. 2011; Park et al. 2012; Woo et al. 2013). We measure the slope of the relation between the virial product, $M_{\mathrm{vir}}$, and $\sigma_{*}$ :

$$
\log M_{\mathrm{vir}}=\alpha+\beta \log \left(\frac{\sigma_{*}}{200 \mathrm{kms}^{-1}}\right)
$$

with our updated AGN sample. We use the traditional forward regression for our calculation: We consider $\sigma_{*}$ as the independent variable and $M_{\mathrm{BH}}$ the dependent variable. Using the FITEXY algorithm (Press et al. 1992) to fit the AGN relation, we obtain $\beta=5.04 \pm 0.19$. This slope is steeper than found in previous studies (e.g., Woo et al. 2013, who report $\beta=3.46 \pm 0.61$ ), and is slightly flatter than but consistent with the most recent measurement of the slope in quiescent galaxies of $5.31 \pm$ 0.33 (Woo et al. 2013). We then use FITEXY to determine the $\langle f\rangle$ necessary to place the AGN sample on the same $M_{\mathrm{BH}}-\sigma_{*}$ relation as quiescent galaxies. Using our updated AGN sample, we obtain $\log \langle f\rangle=0.63 \pm 0.11$, corresponding to $\langle f\rangle=4.31 \pm 1.05$. This number is slightly lower than, but consistent with, the recent values found by Park et al. (2012) and Woo et al. (2013) and is in closer agreement with that found by Graham et al. (2011) when using a forward regression analysis. However, Graham et al. (2011) also raised the issue of a potential sample selection bias in the $M_{\mathrm{BH}}-\sigma_{*}$ relation specifically, there are no $\sigma_{*}$ measurements in objects with $M_{\mathrm{BH}}<10^{6} M_{\odot}$, causing a bias against low- $M_{\mathrm{BH}}$ systems. They use an inverse regression approach to avoid this selection bias. We prefer a forward regression because the $M_{\mathrm{BH}}-\sigma_{*}$ relation is typically used to determine $M_{\mathrm{BH}}$ in galaxies with measured $\sigma_{*}$.

We use our new value of $\langle f\rangle=4.31$ to transform the virial products of the AGN sample to $M_{\mathrm{BH}}$. We place our four objects with successful $\sigma_{*}$ measurements on the $M_{\mathrm{BH}^{-}} \sigma_{*}$ relation in Figure 5, with the rest of the AGN sample shown for comparison. We also show the most recent quiescent $M_{\mathrm{BH}}-\sigma_{*}$ relation from Woo et al. (2013). We see that all four of our objects lie within the expected scatter of the relation, and see no evidence for an offset in the objects at the high end of the relation. Because we were unable to obtain $\sigma_{*}$ measurements for our highest$M_{\mathrm{BH}}$ objects, we fall short of our original goal of densely populating the very high end of the $M_{\mathrm{BH}}-\sigma_{*}$ relation, though we do increase the sample of objects with $10^{8}$ $M_{\odot}<M_{\mathrm{BH}}<10^{9} M_{\odot}$. As noted in previous studies, the AGN sample is still biased towards objects with relatively lower masses than the majority of the quiescent galaxies with dynamical mass measurements.

A morphological or environmental dependence of the $M_{\mathrm{BH}}-\sigma_{*}$ relation could have important implications on the use of a single mean $f$ factor to transform the virial product, $M_{\mathrm{vir}}$, into $M_{\mathrm{BH}}$ in AGNs. To test whether or

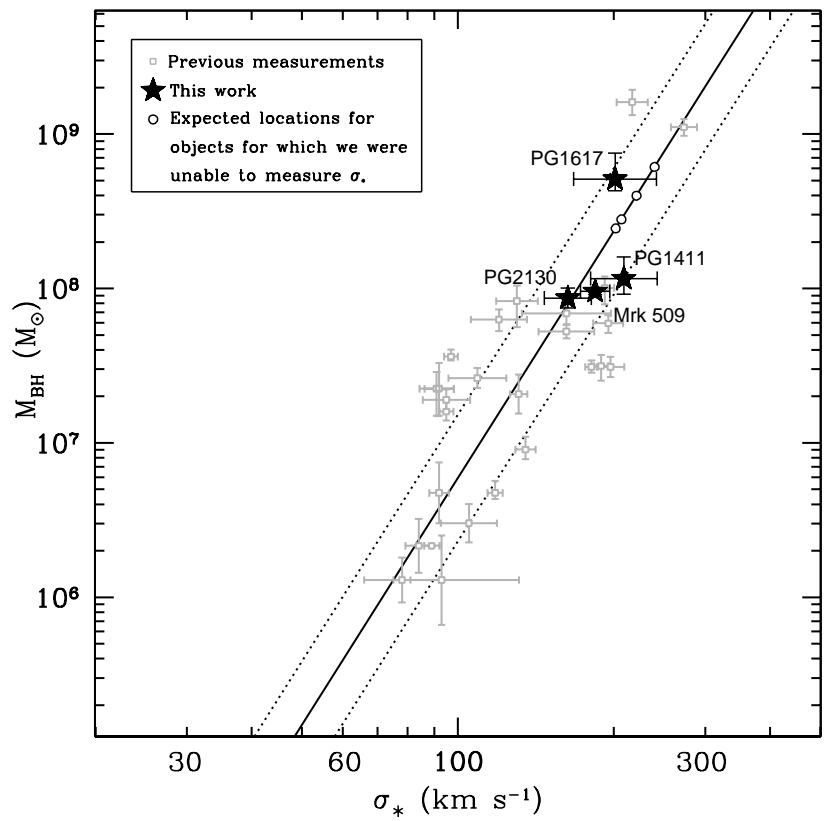

FIG. 5.- The $M_{\mathrm{BH}}-\sigma_{*}$ relation. The gray open squares are AGNs with previous measurements. The sample is composed of the compilation by Woo et al. 2010 and updated by Park et al. 2012 and Woo et al. 2013, with additional updates as described in the text (see Table 6). All $M_{\mathrm{BH}}$ were calculated with our measurement of $f=4.31$. The solid black stars show our new $\sigma_{*}$ measurements. The solid black line shows the most recent measurement of the $M_{\mathrm{BH}}-\sigma_{*}$ relation in quiescent galaxies from Woo et al. 2013, and the dotted lines show the intrinsic scatter of the quiescent galaxies measured by Woo et al. 2013. The open circles denote the expected locations of the objects for which we were unable to measure $\sigma_{*}$ based the $M_{\mathrm{BH}}-\sigma_{*}$ relation.

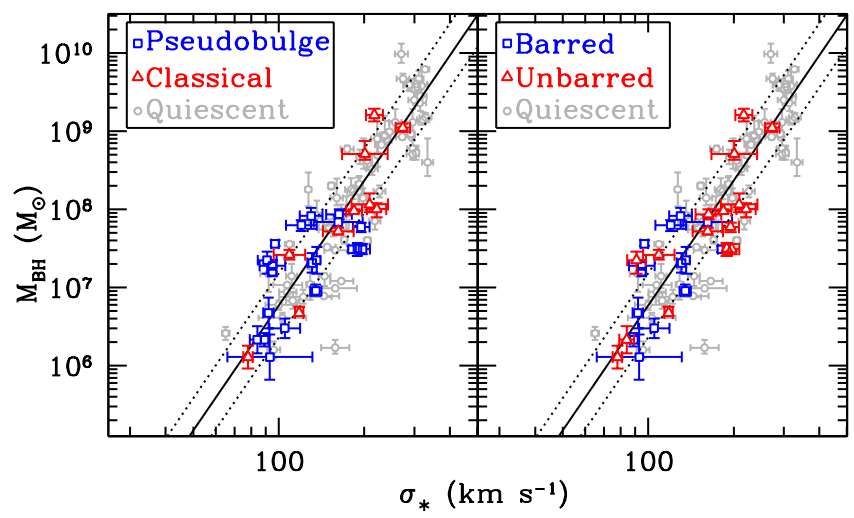

FIG. 6. - The $M_{\mathrm{BH}}-\sigma_{*}$ relation for the same updated AGN sample shown in Figure 5. The quiescent galaxy sample of 72 galaxies from McConnell \& Ma (2013), with updates from Woo et al. (2013), is shown in gray for comparison. The solid black line shows the most recent measurement of the $M_{\mathrm{BH}}-\sigma_{*}$ relation in these quiescent galaxies from Woo et al. 2013, and the dotted lines show their measured intrinsic scatter in the quiescent relation. The left panel shows the AGN sample divided by the type of bulge (pseudobulge or classical), and the right panel shows the AGN sample sorted by the presence of a bar. All $M_{\mathrm{BH}}$ for the AGN sample were calculated with our measurement of $f=4.31$. 
not the AGN sample shows any morphology-dependent effects, we divided our AGN sample into different groups to see if there was any visible offset or change in slope in the $M_{\mathrm{BH}}-\sigma_{*}$ relation. In the left panel of Figure 6 , we divide the AGN sample into two groups: those suspected of hosting a pseudobulge, and those thought to host a classical spheroid at their centers. In the right panel, we divide the sample based on whether or not a central bar is observed. We classified the galaxies using the host-galaxy decompositions from Bentz et al. (2009a) (see their Table 4). Following Kormendy \& Kennicutt (2004), the galaxies were inspected for the presence of the following pseudobulge indicators: flattened bulge morphology, the presence of a nuclear bar, a low $(n<2)$ Sersic index, and the presence of copious dust and star formation in the nucleus without any signature of an ongoing merger. None of these indicators by themselves constitute sufficient evidence that a bulge is a pseudobulge. Therefore, we assumed that galaxies with most of these indicators host a pseudobulge, and galaxies with few or none of these indicators host a classical bulge. Our classifications are listed in Table 6 . It should be noted that host galaxy classifications are particularly difficult to make in high-luminosity quasar hosts, and some galaxies in our sample show signs of disturbed morphologies, so our classifications are somewhat uncertain.

We do not see any signs of an offset in either the galaxies hosting pseudobulges or the galaxies with central bars, unlike several studies that were previously noted in our introduction. If we determine $\langle f\rangle$ with our samples of barred and unbarred galaxies separately, we obtain a result similar to that of Graham et al. (2011). Namely, the galaxies with bars in them yield a lower value $(\langle f\rangle=3.07 \pm 1.00)$ than the galaxies without central bars $(\langle f\rangle=5.92 \pm 1.80)$. However, the barred sample lacks any objects with $M_{\mathrm{BH}}>10^{8} M_{\odot}$ and similarly, the unbarred sample has only three objects with $M_{\mathrm{BH}}$ below $10^{7} M_{\odot}$. Observations of the megamaser sample by Greene et al. (2010) show that about half of their host galaxies (with $M_{\mathrm{BH}}<10^{8} M_{\odot}$ ) fall below the $M_{\mathrm{BH}}-\sigma_{*}$ relation, which again demonstrates that different samples yield different results. In addition, Woo et al. (2010, 2013) and this work have demonstrated that extending the sample to cover the entire $M_{\mathrm{BH}}$ range can significantly impact the measured slopes and mean virial factor, and as such we conclude the difference seen in our samples is not statistically significant due to the systematic uncertainties associated with the lack of a substantial dynamic range in $M_{\mathrm{BH}}$.

\section{SUMMARY}

We measured $\sigma_{*}$ in four quasars at the high-mass end of the AGN $M_{\mathrm{BH}^{-}} \sigma_{*}$ distribution. The measurements of Mrk 509 and PG 1411+442 are the first ever $\sigma_{*}$ measurements for these targets, and the measurements for PG $1617+175$ and PG 2130+099 are updates with improved precision. We were unable to measure $\sigma_{*}$ in the spectra of our highest-redshift objects due to substantial contamination of the host-galaxy spectra by the quasar nucleus and poor sky line subtraction. Future measurements of $\sigma_{*}$ for high-luminosity QSOs will require improved AO technology, substantially longer integration times, or JWST. We also updated the virial products for the AGN $M_{\mathrm{BH}}-\sigma_{*}$ sample with recalculated time lags from the SPEAR method of $\mathrm{Zu}$ et al. (2011) and recalculated the mean virial factor $\langle f\rangle$ used to calibrate the AGN $M_{\mathrm{BH}}$ scale. We obtained $\log \langle f\rangle=0.63 \pm 0.11$, corresponding to $\langle f\rangle=4.31 \pm 1.05$. This is consistent with previous results based on forward regression methods. With our new $\sigma_{*}$ measurements, all four of our objects fall within the expected scatter of the quiescent $M_{\mathrm{BH}}-\sigma_{*}$ relation. We find no evidence in AGNs for a morphology-based deviation from the standard quiescent $M_{\mathrm{BH}}-\sigma_{*}$ relation.

The authors would like to thank Jenny Greene and Alister Graham for their helpful suggestions that improved this work. C.J.G. would like to thank Gisella De Rosa for help with some of the code used in this analysis. B.M.P., R.W.P., and C.J.G. gratefully acknowledge the support of the National Science Foundation through grant AST-1008882 to The Ohio State University. C.J.G. is also supported by a Presidential Fellowship at The Ohio State University. P.M. is grateful for support from the sabbatical visitor program at the North American ALMA Science Center (NAASC) at NRAO and the hospitality of both the NAASC and the University of Virginia while this work was completed. This research has made use of the NASA/IPAC Extragalactic Database (NED) which is operated by the Jet Propulsion Laboratory, California Institute of Technology, under contract with the National Aeronautics and Space Administration. This work is based on observations obtained at the Gemini Observatory, which is operated by the Association of Universities for Research in Astronomy, Inc., under a cooperative agreement with the NSF on behalf of the Gemini partnership: the National Science Foundation (United States), the National Research Council (Canada), CONICYT (Chile), the Australian Research Council (Australia), Ministério da Ciência, Tecnologia e Inovação (Brazil) and Ministerio de Ciencia, Tecnología e Innovación Productiva (Argentina).

\section{REFERENCES}

Barth, A. J., et al. 2011, ApJ, 743, L4

Bentz, M. C., Peterson, B. M., Netzer, H., Pogge, R. W., \& Vestergaard, M. 2009a, ApJ, 697, 160

Bentz, M. C., Peterson, B. M., Pogge, R. W., \& Vestergaard, M. 2009b, ApJ, 694, L166

Bentz, M. C., Peterson, B. M., Pogge, R. W., Vestergaard, M., \& Onken, C. A. 2006, ApJ, 644, 133

Bentz, M. C., et al. 2009c, ApJ, 705, 199

-. 2010, ApJ, 720, L46

-. 2013, ApJ, 767, 149
Boyle, B. J., Georgantopoulos, I., Blair, A. J., Stewart, G. C., Griffiths, R. E., Shanks, T., Gunn, K. F., \& Almaini, O. 1998, MNRAS, 296, 1

Brewer, B. J., et al. 2011, ApJ, 733, L33

Cappellari, M., \& Emsellem, E. 2004, PASP, 116, 138

Christou, J. C., Neichel, B., Rigaut, F., Sheehan, M., McDermid, R. M., \& Trancho, G. 2010, in Adaptive Optics Systems II, SPIE Proc, 7736, 77361R

Dasyra, K. M., et al. 2007, ApJ, 657, 102

Denney, K. D., et al. 2006, ApJ, 653, 152

—. 2010, ApJ, 721, 715 
Di Matteo, T., Colberg, J., Springel, V., Hernquist, L., \& Sijacki, D. 2008, ApJ, 676, 33

Di Matteo, T., Springel, V., \& Hernquist, L. 2005, Nature, 433, 604

Dietrich, M., et al. 2012, ApJ, 757, 53

Faber, S. M., \& Jackson, R. E. 1976, ApJ, 204, 668

Fabian, A. C. 1999, MNRAS, 308, L39

Ferrarese, L., \& Merritt, D. 2000, ApJ, 539, L9

Ferrarese, L., Pogge, R. W., Peterson, B. M., Merritt, D.,

Wandel, A., \& Joseph, C. L. 2001, ApJ, 555, L79

Franceschini, A., Hasinger, G., Miyaji, T., \& Malquori, D. 1999 MNRAS, 310, L5

Gadotti, D. A., \& Kauffmann, G. 2009, MNRAS, 399, 621

Gaskell, C. M., \& Peterson, B. M. 1987, ApJS, 65, 1

Gebhardt, K., et al. 2000a, ApJ, 539, L13

-. 2000b, ApJ, 543, L5

Graham, A. W. 2008a, ApJ, 680, 143

—. 2008b, PASA, 25, 167

Graham, A. W., \& Li, I.-h. 2009, ApJ, 698, 812

Graham, A. W., Onken, C. A., Athanassoula, E., \& Combes, F. 2011, MNRAS, 412, 2211

Greene, J. E., et al. 2010, ApJ, 721, 26

Grier, C. J., et al. 2012, ApJ, 755, 60

-. 2013, ApJ, 764, 47

Gültekin, K., et al. 2009, ApJ, 698, 198

$\mathrm{Hu}$, J. 2008, MNRAS, 386, 2242

Jiang, Y.-F., Greene, J. E., Ho, L. C., Xiao, T., \& Barth, A. J. 2011, ApJ, 742, 68

Jorgensen, I., Franx, M., \& Kjaergaard, P. 1995, MNRAS, 276, 1341

Kauffmann, G., et al. 2003, MNRAS, 346, 1055

Kelly, B. C., Bechtold, J., \& Siemiginowska, A. 2009, ApJ, 698, 895

King, A. 2003, ApJ, 596, L27

-. 2005, ApJ, 635, L121

King, A. R. 2010, MNRAS, 402, 1516

Kinney, A. L., Calzetti, D., Bohlin, R. C., McQuade, K., Storchi-Bergmann, T., \& Schmitt, H. R. 1996, ApJ, 467, 38

Kormendy, J., Bender, R., \& Cornell, M. E. 2011, Nature, 469, 374

Kormendy, J., \& Kennicutt, Jr., R. C. 2004, ARA\&A, 42, 603

Kozłowski, S., et al. 2010, ApJ, 708, 927

Lauer, T. R., Tremaine, S., Richstone, D., \& Faber, S. M. 2007, ApJ, 670, 249

MacLeod, C. L., et al. 2010, ApJ, 721, 1014
Mathur, S., Fields, D., Peterson, B. M., \& Grupe, D. 2012, ApJ, 754,146

McConnell, N. J., \& Ma, C.-P. 2013, ApJ, 764, 184

McConnell, N. J., Ma, C.-P., Gebhardt, K., Wright, S. A.,

Murphy, J. D., Lauer, T. R., Graham, J. R., \& Richstone, D. O. 2011, Nature, 480, 215

Merloni, A., Rudnick, G., \& Di Matteo, T. 2004, MNRAS, 354, L37

Murray, N., Quataert, E., \& Thompson, T. A. 2005, ApJ, 618, 569

Nelson, C. H., Green, R. F., Bower, G., Gebhardt, K., \& Weistrop, D. 2004, ApJ, 615, 652

Nelson, C. H., \& Whittle, M. 1995, ApJS, 99, 67

Onken, C. A., Ferrarese, L., Merritt, D., Peterson, B. M., Pogge,

R. W., Vestergaard, M., \& Wandel, A. 2004, ApJ, 615, 645

Pancoast, A., et al. 2012, ApJ, 754, 49

Park, D., Kelly, B. C., Woo, J.-H., \& Treu, T. 2012, ApJS, 203, 6

Peterson, B. M., et al. 2004, ApJ, 613, 682

Press, W. H., Teukolsky, S. A., Vetterling, W. T., \& Flannery, B. P. 1992, Numerical recipes in FORTRAN. The art of scientific computing (Cambridge: University Press, 2nd ed.), 660

Sanders, D. B., Soifer, B. T., Elias, J. H., Madore, B. F., Matthews, K., Neugebauer, G., \& Scoville, N. Z. 1988, ApJ, 325,74

Silk, J., \& Rees, M. J. 1998, A\&A, 331, L1

Silverman, J. D., et al. 2008, ApJ, 675, 1025

Tody, D. 1986, in Society of Photo-Optical Instrumentation Engineers (SPIE) Conference Series, Vol. 627, Society of Photo-Optical Instrumentation Engineers (SPIE) Conference Series, ed. D. L. Crawford, 733

Tremaine, S., et al. 2002, ApJ, 574, 740

Vacca, W. D., Cushing, M. C., \& Rayner, J. T. 2003, PASP, 115, 389

Veilleux, S., et al. 2009, ApJS, 182, 628

Watson, L. C., Martini, P., Dasyra, K. M., Bentz, M. C.,

Ferrarese, L., Peterson, B. M., Pogge, R. W., \& Tacconi, L. J. 2008, ApJ, 682, L21

Woo, J.-H., Schulze, A., Park, D., Kang, W.-R., Kim, S. C., \& Riechers, D. 2013, arXiv:1305.2946

Woo, J.-H., et al. 2010, ApJ, 716, 269

Yu, Q., \& Tremaine, S. 2002, MNRAS, 335, 965

Zu, Y., Kochanek, C. S., Kozłowski, S., \& Udalski, A. 2013, ApJ, 765,106

Zu, Y., Kochanek, C. S., \& Peterson, B. M. 2011, ApJ, 735, 80

Zubovas, K., \& King, A. R. 2012, MNRAS, 426, 2751 
TABLE 1

Quasar Properties

\begin{tabular}{lccc}
\hline \hline Galaxy & RA & DEC & $z$ \\
$(\mathrm{~J} 2000)$ & $(\mathrm{J} 2000)$ & 0.142 \\
$(\mathrm{NED})$ & +131603 & 0.154 \\
\hline PG 0026+129 & 002913.6 & +252538 & 0.158 \\
PG 0052+251 & 005425.1 & +020309 & 0.089 \\
PG 1226+023 & 122906.7 & +440014 & 0.112 \\
PG 1411+442 & 141348.3 & +172428 & 0.292 \\
PG 1617+175 & 162011.3 & -104320 & 0.034 \\
Mrk 509 & 170124.8 & +100819 & 0.063 \\
PG 2130+099 & 204409.7 & 213227.8 & \\
\hline
\end{tabular}

TABLE 2

Most Prominent Stellar Absorption Features

\begin{tabular}{lr}
\hline \hline Feature & $\begin{array}{c}\text { Rest-frame } \\
\text { Wavelength }(\mu \mathrm{m})\end{array}$ \\
\hline $\mathrm{Mg} \mathrm{I}$ & 1.4880 \\
$\mathrm{Mg} \mathrm{I}$ & 1.5030 \\
$\mathrm{CO}(3-0)$ & 1.5580 \\
$\mathrm{CO}(4-1)$ & 1.5780 \\
$\mathrm{Si}$ & 1.5890 \\
$\mathrm{CO}(5-2)$ & 1.5980 \\
$\mathrm{CO}(6-3)$ & 1.6190 \\
$\mathrm{CO}(8-5)$ & 1.6610 \\
$\mathrm{CO}(9-6)$ & 1.6840 \\
$\mathrm{CO}(10-7)$ & 1.7060 \\
\hline
\end{tabular}

TABLE 3

OBSERVATIONS

\begin{tabular}{cccc}
\hline \hline Target & $\begin{array}{c}\text { Observing } \\
\text { Semester }\end{array}$ & $\begin{array}{c}\text { On-source } \\
\text { Integration time } \\
\text { (hours) }\end{array}$ & Band \\
\hline PG 0026+129 & 2008B & 1.33 & $\mathrm{H}$ \\
PG 0052+251 & $2010 \mathrm{~B}$ & 1.33 & $\mathrm{H}$ \\
PG 1226+023 & $2010 \mathrm{~A}$ & 0.83 & $\mathrm{H}$ \\
PG 1411+442 & $2010 \mathrm{~A}$ & 1.33 & $\mathrm{H}$ \\
PG 1617+175 & $2010 \mathrm{~A}$ & 2.50 & $\mathrm{~K}$ \\
PG 1700+518 & $2010 \mathrm{~A}$ & 3.00 & $\mathrm{H}$ \\
MG 509 $2130+099$ & $2008 \mathrm{~B}$ & 2.00 & $\mathrm{H}$ \\
\hline
\end{tabular}

TABLE 4

Extraction Windows and Measurements

\begin{tabular}{|c|c|c|c|c|c|c|c|c|}
\hline Galaxy & $\begin{array}{c}R_{\text {inner }} \\
(\operatorname{arcsec})\end{array}$ & $\begin{array}{c}R_{\text {outer }} \\
(\text { arcsec) }\end{array}$ & $\begin{array}{c}\text { Stellar } \\
\text { Template }\end{array}$ & $\begin{array}{c}\sigma_{*} \\
\left(\mathrm{~km} \mathrm{~s}^{-1}\right)\end{array}$ & $\begin{array}{c}\text { Best-fit } \\
\chi^{2}\end{array}$ & $D O F$ & $\chi^{2} / D O F$ & $\begin{array}{c}\sigma_{*, \mathrm{e}} \\
\left(\mathrm{km} \mathrm{s}^{-1}\right)\end{array}$ \\
\hline PG 0026+129 & 0.2 & 0.6 & $\ldots$ & $\ldots$ & $\ldots$ & $\cdots$ & $\ldots$ & $\ldots$ \\
\hline PG $0052+251$ & 0.2 & 0.7 & $\cdots$ & $\cdots$ & $\cdots$ & $\cdots$ & $\cdots$ & $\cdots$ \\
\hline PG $1226+023$ & 0.4 & 1.4 & $\ldots$ & $\ldots$ & $\ldots$ & $\ldots$ & $\ldots$ & $\ldots$ \\
\hline PG $1411+442$ & 0.2 & 1.6 & K5 III & $216 \pm 31$ & 858.80 & 914 & 0.940 & $209 \pm 30$ \\
\hline PG $1617+175$ & 0.3 & 1.3 & M5Ia & $201 \pm 37$ & 491.33 & 649 & 0.757 & $201 \pm 37$ \\
\hline PG $1700+518$ & 0.4 & 1.1 & & & & & & \\
\hline Mrk 509 & 0.3 & 1.7 & K5 III & $189 \pm 12$ & 1046.17 & 1134 & 0.923 & $184 \pm 12$ \\
\hline PG 2130+099 & 0.2 & 1.2 & K5 III & $147 \pm 17$ & 548.70 & 914 & 0.600 & $163 \pm 19$ \\
\hline
\end{tabular}

Note. $-R_{\text {inner }}$ and $R_{\text {outer }}$ correspond to the inner and outer radii of the circular extraction annulus for each object. $\chi^{2}$ and $\chi^{2} / D O F$ are reported for the best fits with the K5 III stellar template for PG 1411+442, Mrk 509 and PG 2130+099. For the case of PG 1617, the M5Ia template was used. $R_{\text {inner }}$ and $R_{\text {outer }}$ correspond to the inner and outer radii of the extraction annulus. $\sigma_{*, \text { e }}$ was calculated with the formula for $\mathrm{E}$ and $\mathrm{S} 0$ galaxies from Jorgensen et al. (1995). 
TABLE 5

Reverberation Measurements and Virial Products

\begin{tabular}{|c|c|c|c|c|c|}
\hline Galaxy & $\begin{array}{c}\tau_{\mathrm{H} \beta}{ }^{\mathrm{a}} \\
\text { (days) }\end{array}$ & $\begin{array}{c}\tau_{\mathrm{H} \beta} \\
\text { Reference }\end{array}$ & $\begin{array}{c}\sigma_{\text {line }}(\mathrm{rms}) \\
\left(\mathrm{km} \mathrm{s}^{-1}\right)\end{array}$ & $\begin{array}{l}\sigma_{\text {line }}(\mathrm{rms}) \\
\text { Reference }\end{array}$ & $\begin{array}{c}M_{\mathrm{vir}} \\
\left(10^{6} M_{\odot}\right)\end{array}$ \\
\hline $3 \mathrm{C} 120$ & $27.2_{-1.1}^{+1.1}$ & 1 & $1514 \pm 65$ & 1 & $12.2_{-1.2}^{+1.2}$ \\
\hline 3C 390.3 & $44.3_{-3.3}^{+3.0}$ & 2 & $5455 \pm 278$ & 2 & $260_{-23}^{+36}$ \\
\hline Ark 120 & $35.7_{-9.2}^{+6.7}$ & 3 & $1959 \pm 109$ & 7 & $26.7_{-7.5}^{+5.8}$ \\
\hline Ark 120 & $29.7_{-5.9}^{+3.3}$ & 3 & $1884 \pm 48$ & 7 & $20.6_{-4.2}^{+2.5}$ \\
\hline Arp 151 & $3.6_{-0.2}^{+0.7}$ & 4 & $1252 \pm 46$ & 8,9 & $1.1_{-0.1}^{+0.2}$ \\
\hline Mrk 50 & $10.4_{-0.9}^{+0.8}$ & 5 & $1740 \pm 101$ & 5 & $6.2_{-0.9}^{+0.9}$ \\
\hline Mrk 79 & $25.5_{-14.4}^{+2.9}$ & 3 & $2137 \pm 375$ & 7 & $22.7_{-15.1}^{+8.4}$ \\
\hline Mrk 79 & $30.9_{-2.1}^{+1.4}$ & 3 & $1683 \pm 72$ & 7 & $17.1_{-1.9}^{+1.7^{+}}$ \\
\hline Mrk 79 & $17.2_{-2.2}^{+7.3}$ & 3 & $1854 \pm 72$ & 7 & $11.5_{-1.7}^{+5.0}$ \\
\hline Mrk 79 & $43.6_{-0.8}^{+1.7}$ & 3 & $1883 \pm 246$ & 7 & $30.1_{-7.9}^{+7.9}$ \\
\hline Mrk 110 & $25.3_{-13.1}^{+2.0^{\circ}}$ & 3 & $1196 \pm 141$ & 7 & $7.1_{-4.0}^{+1.8}$ \\
\hline Mrk 110 & $33.9_{-5.3}^{+6.1}$ & 3 & $1115 \pm 103$ & 7 & $8.2_{-2.0}^{+2.1}$ \\
\hline Mrk 110 & $21.5_{-2.1}^{+2.3}$ & 3 & $755 \pm 29$ & 7 & $2.4_{-0.3}^{+0.3}$ \\
\hline Mrk 202 & $3.5_{-0.1}^{+0.1}$ & 4 & $659 \pm 65$ & 8,9 & $0.30_{-0.06}^{+0.06}$ \\
\hline Mrk 279 & $18.3_{-1.1}^{+1.2}$ & 3 & $1420 \pm 96$ & 7 & $7.2_{-1.1}^{+1.1}$ \\
\hline Mrk 509 & $69.9_{-0.3}^{+0.1}$ & 3 & $1276 \pm 28$ & 7 & $22.2_{-1.0}^{+1.0}$ \\
\hline Mrk 590 & $19.0_{-2.6}^{+1.9}$ & 3 & $789 \pm 74$ & 7 & $2.3_{-0.5}^{+0.5}$ \\
\hline Mrk 590 & $19.5_{-4.0}^{+2.0}$ & 3 & $1935 \pm 52$ & 7 & $14.2_{-3.0}^{+1.7}$ \\
\hline Mrk 590 & $32.6_{-8.8}^{+3.5}$ & 3 & $1251 \pm 72$ & 7 & $9.9_{-2.9}^{+1.6}$ \\
\hline Mrk 590 & $30.9_{-2.4}^{+2.5}$ & 3 & $1201 \pm 130$ & 7 & $8.7_{-2.0}^{+2.0}$ \\
\hline Mrk 817 & $20.9_{-2.3}^{+2.3}$ & 3 & $1392 \pm 78$ & 10 & $7.9_{-1.2}^{+1.2}$ \\
\hline Mrk 817 & $17.2_{-2.7}^{+1.9}$ & 3 & $1971 \pm 96$ & 10 & $13.0_{-2.4}^{+1.9}$ \\
\hline Mrk 817 & $35.9_{-5.8}^{+4.8}$ & 3 & $1729 \pm 158$ & 10 & $20.9_{-5.1}^{+4.7}$ \\
\hline Mrk 817 & $10.8_{-1.0}^{+1.5}$ & 3 & $3150 \pm 295$ & 10 & $20.9_{-4.4}^{+4.9}$ \\
\hline Mrk 1310 & $4.2_{-0.1}^{+0.9}$ & 4 & $755 \pm 138$ & 8,9 & $0.5_{-0.2}^{+0.2 .4}$ \\
\hline NGC 3227 & $10.6_{-6.1}^{+6.1}$ & 3 & $1925 \pm 124$ & 10 & $7.7_{-4.5}^{+4.5}$ \\
\hline NGC 3227 & $4.4_{-0.5}^{+0.3}$ & 3 & $2018 \pm 174$ & 10 & $3.5_{-0.7}^{+0.7}$ \\
\hline NGC 3516 & $14.6_{-1.1}^{+1.4}$ & 3 & $1591 \pm 10$ & 10 & $7.2_{-0.6}^{+0.7}$ \\
\hline NGC 3783 & $7.3_{-0.7}^{+0.3}$ & 3 & $1753 \pm 141$ & 7 & $4.4_{-0.8}^{+0.0}$ \\
\hline NGC 4051 & $2.5_{-0.1}^{+0.1}$ & 3 & $1034 \pm 41$ & 10 & $0.5_{-0.1}^{+0.1}$ \\
\hline
\end{tabular}

NOTE. - This table has been abbreviated and can be downloaded from the arXiv in full with the article source files. The published version of the paper will include the full table. References: 1. Grier et al. 2012; 2. Dietrich et al. 2012; 3. Zu et al. 2011; 4. This work; 5. Barth et al. 2011; 6. Grier et al. 2013; 7. Peterson et al. 2004; 8. Bentz et al. 2009b; 9. Park et al. 2012. 10. Denney et al. 2010 11. Bentz et al. 2006; 12. Denney et al. 2006

a Time lags are all given in the rest frame. 
TABLE 6

The AGN $M_{\mathrm{BH}^{-}} \sigma_{*}$ SAmple

\begin{tabular}{|c|c|c|c|c|c|}
\hline Galaxy & Classification $^{\mathrm{a}}$ & $\begin{array}{c}M_{\mathrm{vir}}{ }^{\mathrm{b}} \\
\left(10^{6} M_{\odot}\right)\end{array}$ & $\begin{array}{c}M_{\mathrm{BH}^{\mathrm{c}}} \\
\left(10^{6} M_{\odot}\right)\end{array}$ & $\begin{array}{c}\sigma_{*} \\
\left(\mathrm{~km} \mathrm{~s}^{-1}\right)\end{array}$ & $\begin{array}{c}\sigma_{*} \\
\text { Reference }\end{array}$ \\
\hline $3 \mathrm{C} 120$ & Classical & $12.2_{-1.2}^{+1.2}$ & $52.6_{-5.2}^{+5.2}$ & $162 \pm 20$ & 1 \\
\hline 3C 390.3 & Classical & $260_{-23}^{+36}$ & $1120.6_{-142.2}^{+138.0}$ & $273 \pm 16$ & 2 \\
\hline Ark 120 & Classical & $23.4_{-5.7}^{+4.0}$ & $100.9_{-24.6}^{+17.2}$ & $192 \pm 8$ & 3 \\
\hline Arp 151 & Classical & $1.1_{-0.1}^{+0.2}$ & $4.7_{-0.4}^{+0.9}$ & $118 \pm 4$ & 4 \\
\hline Mrk 50 & Classical & $6.2_{-0.9}^{+0.9}$ & $26.3_{-3.9}^{+3.9}$ & $109 \pm 14$ & 5 \\
\hline Mrk 79 & Barred Pseudobulge & $19.2_{-7.4}^{+4.5}$ & $82.8_{-31.9}^{+19.4}$ & $130 \pm 12$ & 2 \\
\hline Mrk 110 & Pseudobulge & $5.2_{-2.1}^{+1.3}$ & $22.4_{-9.1}^{+5.6}$ & $91 \pm 7$ & 6 \\
\hline Mrk 202 & Classical & $0.3_{-0.1}^{+0.1}$ & $1.3_{-0.4}^{+0.4}$ & $78 \pm 3$ & 4 \\
\hline Mrk 279 & Pseudobulge & $7.2_{-1.1}^{+1.1}$ & $31.0_{-4.7}^{+4.4}$ & $197 \pm 12$ & 2 \\
\hline Mrk 509 & Classical & $22.2_{-1.0}^{+1.0}$ & $95.7_{-4.3}^{+4.3}$ & $184 \pm 12$ & 7 \\
\hline Mrk 590 & Pseudobulge & $7.3_{-1.6}^{+1.2}$ & $31.5_{-6.9}^{+5.3}$ & $189 \pm 6$ & 2 \\
\hline Mrk 817 & Barred Pseudobulge & $14.6_{-2.5}^{+2.0}$ & $\begin{array}{l}62.9_{-10.8}^{+9.5} \\
\end{array}$ & $120 \pm 15$ & 2 \\
\hline Mrk 1310 & Pseudobulge & $0.47_{-0.17}^{+0.20}$ & $2.2_{-0.9}^{+0.9^{\circ}}$ & $84 \pm 5$ & 4 \\
\hline NGC 3227 & Barred Pseudobulge & $5.2_{-2.1}^{+2.0}$ & $22.4_{-9.1}^{+8.6}$ & $92 \pm 6$ & 3 \\
\hline NGC 3516 & Barred Pseudobulge & $7.2_{-0.6}^{+0.7}$ & $31.0_{-2.6}^{+3.0}$ & $181 \pm 5$ & 2 \\
\hline NGC 3783 & Barred Pseudobulge & $4.4_{-0.8}^{+0.0}$ & $19.0_{-3.4}^{+3.0}$ & $95 \pm 10$ & 8 \\
\hline NGC 4051 & Barred Pseudobulge & $0.5_{-0.1}^{+0.8}$ & $2.2_{-0.4}^{+0.4}$ & $89 \pm 3$ & 2 \\
\hline NGC 4151 & Barred Pseudobulge & $8.4_{-0.5}^{+0.9}$ & $36.2_{-2.2}^{+3.4}$ & $97 \pm 3$ & 2 \\
\hline NGC 4253 & Barred Pseudobulge & $0.3_{-0.2}^{+0.2}$ & $1.3_{-0.9}^{+0.9}$ & $93 \pm 32$ & 4 \\
\hline NGC 4593 & Barred Pseudobulge & $2.1_{-0.3}^{+0.4}$ & $9.1_{-1.3}^{+1.7}$ & $135 \pm 6$ & 2 \\
\hline NGC 4748 & Barred Pseudobulge & $0.7_{-0.2}^{+0.3}$ & $3.0_{-0.9}^{+0.9}$ & $105 \pm 13$ & 4 \\
\hline NGC 5548 & Pseudobulge & $13.8_{-2.0}^{+1.7}$ & $59.5_{-8.6}^{+7.3}$ & $195 \pm 13$ & 4 \\
\hline NGC 6814 & Barred Pseudobulge & $3.7_{-0.5}^{+0.5}$ & $15.9_{-2.2}^{+2.6}$ & $95 \pm 3$ & 4 \\
\hline NGC 7469 & Barred Pseudobulge & $4.8_{-1.4}^{+1.4}$ & $20.7_{-6.0}^{+6.2}$ & $131 \pm 5$ & 2 \\
\hline PG $1229+204$ & Barred Pseudobulge & $16.0_{-2.6}^{+2.7}$ & $69.0_{-11.2}^{+11.6}$ & $162 \pm 32$ & 9 \\
\hline PG $1411+442$ & Classical & $26.9_{-6.3}^{+8.7}$ & $115.9_{-27.2}^{+37.5}$ & $209 \pm 30$ & 7 \\
\hline PG $1426+015$ & Classical & $373.3_{-71.6}^{+68.3}$ & $1609_{-309}^{+296}$ & $217 \pm 15$ & 10 \\
\hline PG $1617+175$ & Classical & $118.6_{-20.6}^{+45.8}$ & $511.2_{-88.8}^{+197.4}$ & $201 \pm 37$ & 7 \\
\hline PG 2130+099 & Pseudobulge & $20.1_{-3.0}^{+3.0^{\circ}}$ & $86.6_{-12.9}^{+12.9}$ & $163 \pm 19$ & 7 \\
\hline SBS $1116+583 \mathrm{~A}$ & Barred Pseudobulge & $1.1_{-0.5}^{+0.5}$ & $4.7_{-2.2}^{+2.2}$ & $92 \pm 4$ & 4 \\
\hline
\end{tabular}

Note. - Velocity Dispersion References: 1. Nelson \& Whittle 1995; 2. Nelson et al. 2004; 3. Woo et al. 2013; 4. Woo et al. 2010; 5. Barth et al. 2011; 6. Ferrarese et al. 2001; 7. This work; 8. Onken et al. 2004; 9. Dasyra et al. 2007; 10. Watson et al. 2008.

a Classifications were made using the host galaxy decompositions of Bentz et al. (2009a) and the critera are discussed in the text.

b For objects with multiple RM measurements, the adopted virial product is the average of the logarithm of the different virial products.

${ }^{c} M_{\mathrm{BH}}$ were computed using $f=4.31$. 